\title{
Predicting cause of death from free-text health summaries: development of an interpretable machine learning tool
}

1 Chris McWilliams ${ }^{1 ¥}$, Eleanor I. Walsh ${ }^{2 ¥ *}$, Avon Huxor ${ }^{3}$, Emma L. Turner ${ }^{2}$, Raul Santos-

2 Rodriguez ${ }^{1}$

$3{ }^{1}$ School of Computer Science, Electrical and Electronic Engineering, and Engineering Mathematics,

4 Faculty of Engineering, University of Bristol, Bristol, UK

$5 \quad{ }^{2}$ Bristol Medical School - Population Health Sciences, Faculty of Health Sciences, University of

6 Bristol, Bristol, UK

$7 \quad{ }^{3}$ School of Engineering, Mathematics and Physical Sciences, University of Exeter, Exeter, UK

$8 \stackrel{¥}{ }$ co-lead authors

9 * Correspondence:

10 Corresponding Author

11 e.walsh@bristol.ac.uk

12 Keywords: machine learning, free-text, accountability, transparency, prostate cancer, medical 13 records

\section{Structured Abstract}

15 Purpose: Accurately assigning cause of death is vital to understanding health outcomes in the 16 population and improving health care provision. Cancer-specific cause of death is a key outcome in

17 clinical trials, but assignment of cause of death from death certification is prone to misattribution,

18 therefore can have an impact on cancer-specific trial mortality outcome measures.

Methods: We developed an interpretable machine learning classifier to predict prostate cancer death from free-text summaries of medical history for prostate cancer patients (CAP). We developed visualisations to highlight the predictive elements of the free-text summaries. These were used by the project analysts to gain an insight of how the predictions were made.

Results: Compared to independent human expert assignment, the classifier showed $>90 \%$ accuracy in predicting prostate cancer death in test subset of the CAP dataset. Informal feedback suggested that these visualisations would require adaptation to be useful to clinical experts when assessing the appropriateness of these ML predictions in a clinical setting. Notably, key features used by the classifier to predict prostate cancer death and emphasised in the visualisations, were considered to be clinically important signs of progressing prostate cancer based on prior knowledge of the dataset.

Conclusion: The results suggest that our interpretability approach improve analyst confidence in the tool, and reveal how the approach could be developed to produce a decision-support tool that would be useful to health care reviewers. As such, we have published the code on GitHub to allow others to apply our methodology to their data (https://zenodo.org/badge/latestdoi/294910364). 
medRxiv preprint doi: https://doi.org/10.1101/2021.07.15.21260082; this version posted July 16, 2021. The copyright holder for this preprint (which was not certified by peer review) is the author/funder, who has granted medRxiv a license to display the preprint in perpetuity.

It is made available under a CC-BY 4.0 International license Free-text cause of death predictions

\section{Declarations}

35 Funding: This work was funded by the Jean Golding Institute Seed Corn Fund 2019-2020. The CAP

36 trial was funded by grants from Cancer Research UK (C11043/A4286, C18281/A8145,

37 C18281/A11326, C18281/A15064, C18281/A24432). The UK Department of Health National

38 Institute of Health Research provided partial funding. CM is funded by the HDR UK South West

39 Better Care Partnership. RSR is supported by the UKRI Turing AI Fellowship EP/V024817/1.

40 Conflict of Interest: The authors declare that the research was conducted in the absence of any

Availability of data: The dataset analysed in this studied cannot be shared publicly for reasons of 43 data protection.

Code availability: Code is available at https://zenodo.org/badge/latestdoi/294910364

Author Contributions: $\mathrm{CM}$ and $\mathrm{AH}$ conducted data analysis and modelling. EW, AH, ET and RS developed the study concept and design. EW and ET contributed domain expertise and detailed understanding of the dataset. RS contributed expertise in interpretable machine learning. EW and CM wrote the manuscript and all authors contributed edits and revisions.

Acknowledgments: We acknowledge the contribution of the CAP trial group, including the summaries written by the fieldworkers Naomi Williams, Siaw Yein Ng, Laura Hughes, Elizabeth Hill, Charlotte Davies, Liz Salter, Jainnee Mauree, Mari-Anne Rowlands, Leah Bowen, Sean Harrison, Pete Holding and Lindsey Bell. And thank you to Tyler Seibert, Assistant Professor and Radiologist at University California San Diego for his insights into the data visualisation outputs from this project. The CAP trial recognises that this work uses data provided by patients and collected by the NHS as part of their care and support. We also acknowledge the work of the NHS Digital Organization and Office of National Statistics for their assistance with this study.

Ethics statement: This work was completed within the ethical approval already held CAP project (Derby National Research Ethics Service Committee East Midlands \& NHS Health Research Authority Confidential Advisory Group (CAG) approvals). In the case of the CAP trial, the names, hospital identifiers, full dates etc.). A derived extract of the, already pseudonymised, CAP summaries were then analysed.

66 Consent to participate: Not applicable.

Consent to publish: Not applicable. 
medRxiv preprint doi: https://doi.org/10.1101/2021.07.15.21260082; this version posted July 16, 2021. The copyright holder for this preprint

(which was not certified by peer review) is the author/funder, who has granted medRxiv a license to display the preprint in perpetuity.

It is made available under a CC-BY 4.0 International license Free-text cause of death predictions

\section{Introduction}

Free-text electronic health records (hereafter, health records) contain information about a patient's medical history. In health care, it is common for human experts to review health records to inform decision making and clinical practice. This review process can be time consuming and prone to error and there is considerable potential for algorithmic methods to support human decision-making in this context (1). One example of this process is retrospective auditing of health care practice, which often requires trained experts to manually review and assign outcomes or labels to the cases reviewed. The high cost in person-hours can make such studies prohibitively expensive, including clinical trials, which are essential for improving the delivery of patient care.

As one of the most common cancers diagnosed in the UK, it is not surprising that prostate cancer is one of the cancers on which text mining of health records for research has focused (2). Much of this work has concentrated on predicting cancer detection (3), disease progression (4) and optimizing treatment (5). Knowledge of underlying cause of death is another key health outcome and machine learning techniques have made it possible to extract cancer mortality directly from Medical Certificates of Cause of Death (MCCD)(6). But these methods have not taken into account the inherent misattribution that exists within the death certificate. For example, prostate cancer deaths can be misattributed to other causes which result in underestimates of prostate cancer as a cause of death. This is the case when deaths are attributed to the complications from investigations or treatment of prostate cancer, rather than the disease itself (e.g. infection from biopsy, post-surgery complications). Deaths from other causes can also be attributed to prostate cancer which would cause an over-estimate of prostate cancer as cause of death (7). This was demonstrated in the Cluster randomised trial of PSA testing for prostate cancer (CAP) where an independent committee assigned cause of death, finding that death certification produced false positive prostate cancer deaths $9 \%$ of the time. This increased to $23 \%$ if the individual had another cancer (not prostate cancer) diagnosed during their lifetime (8). It led to a recommendation that assignment of prostate cancer death, especially as an outcome in trials research, should be confirmed by an independent expert committee (8). In the CAP trial, semi-structured free-text summaries of a patient's medical history from hospital records are created by trained fieldworkers, which are then reviewed by an independent committee of experts and assigned as either prostate cancer related death or not. The CAP dataset thus provides a binary classification task - the identification of prostate cancer death - for which machine learning algorithms can be trained on the annotation of human experts.

There have been significant advances in the field of text mining in recent years, with general purpose deep neural network models such as BERT (9) achieving state-of-the art performance on multiple natural language processing (NLP) tasks, such as question answering and named entity recognition. Applying such NLP methodologies to clinical text data presents various challenges (10) and perhaps the most significant is the shift in word distributions as compared to the standard corpora on which models are trained. This challenge can be overcome by training on general biomedical corpora (11) and/or by developing task-specific models. For example, the state-of-the-art in the detection of medical concepts, such as ICD-10 codes, is to use task-specific recurrent neural network architectures $(12,13)$ which are pre-trained on a medical corpus such as MIMIC (14). However, on specific tasks, classical NLP approaches are still able to compete with deep learning methods (15-17).

This work addresses the task of document classification. An extensive review of this topic, in relation to clinical text data, is provided by Mujtaba et al (10). Specifically, we aim to train a binary classifier on human expert annotations in order to identify patients that died from prostate cancer using the CAP health records described above. Although optimal performance in such tasks is likely 
medRxiv preprint doi: https://doi.org/10.1101/2021.07.15.21260082; this version posted July 16, 2021. The copyright holder for this preprint (which was not certified by peer review) is the author/funder, who has granted medRxiv a license to display the preprint in perpetuity. It is made available under a CC-BY 4.0 International license Fee-text cause of death predictions

114 to be achieved by deep learning models, we are more concerned here with the interpretability of the

115 classifier in the context of decision-support. Interpretability is key to the safe and effective

116 deployment of machine learning (ML) in contexts where there is a human-in-the-loop (18). The

117 related concepts of fairness (19), accountability (20) and transparency (21) have become key tenets

118 of ethical artificial intelligence and are nowhere more relevant than in the medical domain. Human

119 users of an ML algorithm must be able to engage with and understand its predictions. Crucially, the

120 user must have the confidence to either accept or reject the predictions based on their clinical

121 expertise and a clear understanding of how the predictions were made.

122 In this study we developed graphical methods to explain which textual elements contribute to the

123 classification of a health records from CAP by a machine learning algorithm. We also used methods

124 from the FAT Forensics toolbox (22) and the TreeInterpreter Python package (23) to quantify feature

125 contributions. These contributions were then displayed to the user in an interpretable and visually

126 engaging format. The classifiers and interpretability methods were developed for the CAP dataset,

127 using expert committee assignment of prostate cancer-related death to train the classifiers. However,

128 the intention is that these methods will be developed into a decision-support tool that would help

129 human experts in the time-consuming task of classification of health records, both for this specific

130 task and for similar tasks with different data sources.

\section{2. Materials and Methods}

132 The machine learning classifier was developed using the CAP dataset, following which we

133 investigated a variety of visualization techniques to aid interpretation of the ML predictions. The

134 CAP dataset is outlined below (2.1). All code was written in Python and has been released publicly at

135 https://zenodo.org/badge/latestdoi/294910364 to facilitate re-use by other groups working in this

136 area.

\section{$137 \quad$ 2.1 CAP medical history summaries}

138 The CAP trial is a cluster-randomised control trial (RCT) that aims to investigate the effectiveness of 139 screening for prostate cancer in the UK population, which has been running since 2001. The CAP

140 dataset used in this study contains 2,606 medical summaries of men who had a diagnosis of prostate

141 cancer, registered with the UK National Cancer Registrations Service in England and Wales, and

142 who had died by $31^{\text {st }}$ March 2016 (see Table 1 for characteristics). Deaths were notified by the Office

143 of National Statistics who provide death certification information via NHS Digital.

144 The medical summaries were written by 13 trained fieldworkers who extracted prostate cancer and 145 end of life information directly from the medical records in hospitals. The semi-structured summaries 146 of the patients' medical history include information about prostate cancer severity, treatments, and 147 progression, as well as co-morbidities and competing causes of death. The full list of text fields 148 present in this data are given in table ST1 in Online Resource 1.

149 The medical summaries have been reviewed by an independent cause of death evaluation committee 150 made up of clinical experts (i.e. urological surgeons, pathologists and oncologists) who decide 151 whether the cause of death is due to prostate cancer for each case, or whether the patient died 'with' 152 prostate cancer and not 'from' prostate cancer. This human made decision was used as the ground 153 truth against which to train the classifier.

Table 1. Characteristics of prostate cancer patients in the CAP dataset

\begin{tabular}{l|l} 
Characteristic & Prostate cancer dataset (CAP)
\end{tabular}

This is a provisional file, not the final typeset article 
medRxiv preprint doi: https://doi.org/10.1101/2021.07.15.21260082; this version posted July 16, 2021. The copyright holder for this preprint (which was not certified by peer review) is the author/funder, who has granted medRxiv a license to display the preprint in perpetuity.

It is made available under a CC-BY 4.0 Internatiopal license

\begin{tabular}{|l|l|}
\hline No. of patients & 2,606 \\
\hline Sex & M: 2,606 (100\%); F: N/A \\
\hline Age at diagnosis (mean) & 67.5 years (IQR: 64.1-71.3) \\
\hline Age at death (mean) & 70.2 years (IQR: 66.5-74.5) \\
\hline Year of death (range) & 2003 - 2016 \\
\hline Primary outcome & $\begin{array}{l}\text { Prostate cancer related death } \\
\text { Yes: } 1,095(42 \%) ; \text { No: } 1,511 \text { (58\%) }\end{array}$ \\
\hline Note types & $\begin{array}{l}\text { Oncology and urology notes, inpatient notes, outpatient notes, } \\
\text { radiology notes, multidisciplinary team meeting notes, lab reports, } \\
\text { operative reports }\end{array}$ \\
\hline
\end{tabular}

\section{$155 \quad 2.2$ Machine learning pipeline}

156 We used a bag-of-words representation of the health records which was produced as follows. The 157 semi-structured text fields were concatenated into a single string and then lemmatized using the 158 WordNetLemmatizer from Python's Natural Language Toolkit (NLTK). The full details of the 159 lemmatization are provided in Algorithm A1 in Online Resource 1. We then extracted features from the lemmatized text using CountVectorizer and TfidfTransformer from Scikit-learn. The hyperparameters of CountVectorizer were optimized using GridSearchCV along with those of the

162 classifier algorithm. The classifier was trained on $80 \%$ of the dataset with $20 \%$ held out for testing. Following optimisation of the hyperparameters with 5-fold cross validation, the best classifier was refitted to the full training data. Full details of the feature extraction and model training are provided in Algorithm A2 in Online Resource 1. During model development we tested three classifier algorithms from scikit-learn. However, the code can easily be adapted to use any off-the-shelf or bespoke classifier. We evaluated classifier performance using a suite of metrics, including accuracy and AUC (area under the curve).

\subsection{Interpretability methods}

170 On top of the classification pipeline, we developed interpretable outputs that allow users to engage with the classifier's prediction and which are intended to act as a proof-of-concept for a future decision support tool based on this work. To achieve interpretability, we focused on the communication of feature importance. We intended to convey an intuition for how the classifier works and to allow users to consider whether they agree with individual predictions based on how that prediction was made. In other words, we introduced the key elements of accountability (20) and trust (18) to the classification system. We used word clouds to visually represent the relative importance of the features (words and bigrams) by scaling their size. The colours of the words were used to display the sign of the contribution of the feature towards the class prediction (where this information was available). We extended this visual representation by producing augmented versions of the original free-text summaries that displayed feature contributions in the context of the original 181 language used.

182 To quantify feature importance, we used four different approaches. Two of these methods are specific to the tree-based classifiers, while two are generally applicable and can be used with any machine learning classifier. The four methods are as follows: 
medRxiv preprint doi: https://doi.org/10.1101/2021.07.15.21260082; this version posted July 16, 2021. The copyright holder for this preprint (which was not certified by peer review) is the author/funder, who has granted medRxiv a license to display the preprint in perpetuity.

aggregate measure of importance (i.e. at the level of the whole dataset). It also does not capture the sign of the contribution of the feature.

2. TreeInterpreter - This package (23) computes feature importance by decomposing a single prediction into a sum over feature contributions. It captures both the sign and magnitude of each feature contribution to a single prediction and can be averaged over many predictions to produce an aggregate measure of importance.

3. LIME - Local Interpretable Model-agnostic Explanations (25) are a standard tool of explainable AI and are implemented in (22). They provide a local measure of feature contribution that can be used with any machine learning classifier.

4. SHAP - SHapley Additive exPlanations (26) is a model agnostic explainer based on game theoretic methods that also provides a local measure of feature importance.

\section{Results}

A random forest classifier proved to be the best performing algorithm for the task of predicting prostate cancer death according to accuracy and F1 score. This model achieved a classification accuracy of $91.8 \%$ and an F1 of 0.931 on the test data. (Its cross-validation performance is illustrated in figure S0 in Online Resource 1.) A support vector classifier and linear regression classifier achieved comparable levels of performance (see Table 2 and Figure 1). We attempted recalibration of the random forest classifier output using both isotonic and sigmoid (27) methods (see Figure S1 in Online Resource 1) but both methods made only minor improvements to the probabilities output. As such, the main results presented here are for the uncalibrated classifiers. We hypothesized that the cases represented in the CAP dataset exhibited different degrees of classification difficulty, corresponding to the method with which the cases had been originally labelled with a cause of death. These different methods are collectively referred to as the "cause of death route" (COD) and are explained in Algorithm A3 in Online Resource 1. Using the COD route, we stratified the dataset into hard and easy cases. We found that the classifier performance was worse for the hard cases than the easy cases (Figure S2 in Online Resource 1) and that the probabilities were better calibrated for the easy cases (Figure S3 in Online Resource 1). For hard cases, the classifiers tended to underestimate the probability of prostate cancer death towards the lower end of the range. This implies the existence of patients who actually died of prostate cancer, but which look to the classifier like they did not. These results suggest that the stratification of cases based on COD route is meaningful and may have significant implication for how a decision support tool could be used and evaluated in the future. However, it should be noted that these groups are imbalanced with 2340 and 270 easy and hard cases appearing in the dataset, respectively. We investigated the effect of authorship and found evidence of clustering based on language style (Figure S4 in Online Resource 1) but this did not significantly affect performance (see section SM1.1 in Online Resource 1).

Table 2. Performance summary of the three classifiers. SVC = Support Vector Classifier; $L R=$ Logistic Regression; $R F=$ Random Forest. Bold face indicates the best performance for each metric.

\begin{tabular}{|c|l|l|l|l|c|l|}
\hline Classifier & AUC & Accuracy & Precision & Recall & F1 & Specificity \\
\hline SVC & $\mathbf{0 . 9 7 4 9}$ & 0.9157 & $\mathbf{0 . 9 3 7 9}$ & 0.9199 & 0.9288 & $\mathbf{0 . 9 0 9 5}$ \\
\hline LR & 0.9733 & 0.9023 & 0.9196 & 0.9167 & 0.9181 & 0.8810 \\
\hline
\end{tabular}


medRxiv preprint doi: https://doi.org/10.1101/2021.07.15.21260082; this version posted July 16, 2021. The copyright holder for this preprint

(which was not certified by peer review) is the author/funder, who has granted medRxiv a license to display the preprint in perpetuity.

It is made available under a CC-BY 4.0 International license Free-text cause of death predictions

\begin{tabular}{|l|l|l|l|l|l|l|}
\hline RF & 0.9731 & $\mathbf{0 . 9 1 7 6}$ & 0.9325 & $\mathbf{0 . 9 2 9 5}$ & $\mathbf{0 . 9 3 1 0}$ & 0.9000 \\
\hline
\end{tabular}

The random forest was chosen to explore interpretability based on its performance. This choice also allowed us to explore feature importance metrics that are only applicable to tree-based algorithms. We compared feature rankings obtained using the four metrics for feature importance using Spearman's rank correlation coefficient (see table ST2 in Online Resource 1). The rankings according to SHAP, LIME and Gini importance were all moderately to strongly correlated with the TreeInterpreter rankings $(\rho=0.54-0.65)$. Here we present interpretability results using the

232 TreeInterpreter metric for feature importance, but the equivalent outputs can easily be produced using the alternative metrics (see for example, Figures S5 and S6 in Online Resource 1).

We produced word clouds to illustrate the most important features that contributed to the classifier predictions. These word clouds were shared with members of the CAP study team, who confirmed that the classifier was using clinically meaningful information to make predictions. Features with large contributions (such as "bone scan", "spine", "hormone", "androgen") tend to be associated with advanced stage prostate cancer. These features can contribute positively or negatively to the classification depending on the frequency of occurrence of the term in the health record. For example, both "hormone" and "bone scan" contribute positively to the classification of prostate cancer death when present in individual cases (see Figure 2(B)) but when averaged across the dataset they are indicative of non-prostate cancer death (see Figure 2(A)).

243 Therefore, we felt it was necessary to see these feature contributions in the context of the original text, to determine if the classifier is correctly identifying textual elements that indicate prostate cancer death. For this reason, we sought a format that would allow users to engage with the classifier output and could potentially be used for decision support. The solution, arrived through dialogue with members of the CAP project, was to produce augmented versions of the original health records which we refer to as interpretable vignettes. A partial view of one of the interpretable vignettes is shown in figure 3 and full examples are provided in Online Resources 2 and 3. The text uses the same formatting as the word clouds to show feature contributions. Here the reader can see the context in which the features appear and can therefore use their judgement to determine if the classifier is using the textual element in a meaningful way towards its prediction (28). A legend is provided so that readers can interpret the relative feature contribution sizes and summary information is provided in the header of the vignette.

\section{Discussion}

For a machine learning tool to be useful, especially in the medical domain, it is essential for the user to be able to interpret its output. As such, it is common for studies about clinical decision support or prediction modelling to include a discussion of interpretability (29-31). However, as Lipton points out, "interpretability is not a monolithic concept" (32) - it includes distinct yet intersecting ideas such as comprehension, transparency and trust. It is also subjective, in that what one user may find interpretable another user may not. Most importantly, what the data scientist may consider to be interpretable is not necessarily of use to a clinical decision maker. In this study we used four standard interpretability metrics to produce measures of feature importance from a machine learning classifier that was trained to identify prostate cancer deaths from medical summaries. We then developed visual representations of these feature importances and presented them to one of the trained CAP 
medRxiv preprint doi: https://doi.org/10.1101/2021.07.15.21260082; this version posted July 16, 2021. The copyright holder for this preprint

(which was not certified by peer review) is the author/funder, who has granted medRxiv a license to display the preprint in perpetuity.

It is made available under a CC-BY 4.0 International license Fee-text cause of death predictions

reviewers, who would be the intended users of a future decision support system based on this work. Their feedback is summarised below and used to highlight the strengths and weaknesses of our approach, and to identify directions for future work.

The word clouds of feature contributions were intended to provide a high-level overview of what the classifier learned from the data. The reviewer showed a preference for an alternative presentation of this information, stating that "they may be a reasonable way to display the model weights, but I probably would better understand a sorted list in tabular form with associated weight magnitudes". The augmented vignettes (Online Resources 2 and 3) proved to be more useful. These allowed the reviewer to engage with individual predictions, by highlighting features in the original text, using font colour and size to indicate the direction and magnitude of the contribution to the prediction. Similar visualisations have been used in other ML studies to provide interpretability $(28,33-35)$. Being able to see the feature contributions in the semantic context of the original text enabled the reviewer to determine where the classifier was correctly or incorrectly using features. In general, the reviewer felt that the highlighted feature contributions were consistent with their clinical assessment:

"Many of the colors make sense here (in Online Resource 2). Metastases would be consistent with prostate cancer death...Meanwhile, mentions of "lungs", "ascites", "stomach", and "thorax" in the vignette suggest the patient has some non-prostate-cancer condition that is worthy of attention - those are appropriately yellow."

The features appearing in Online Resources 2 and 3 which were associated with prostate cancer death, aligned with the indicators of advancing disease (e.g. bony metastases, hormone treatment) that are used as clinical outcomes in prostate cancer trials (36). However, the feedback made it clear that the visualisations were less interpretable than intended:

'I am confused, again, that 'bone scan' and 'hormone' are blue here (in Online Resource 3) but were each yellow elsewhere (bone scan was yellow for Online Resource 2 and in Figure $2 A$; hormone was yellow in Figure 2A)."

Here the reviewer is referring to the ability of a feature (e.g. 'bone scan') to contribute positively to a classification of prostate cancer death when present in the text, but to contribute negatively to the classification when it is absent. This is an example of the potential for conflict, referred to by Lipton (32), between what is a transparent and faithful representation of the mechanism of a classifier and what is easily understandable by a human user. In this case, the problem might be overcome either by including some indication of the actual feature value or by providing some training to the user to resolve the apparent inconsistency.

The augmented vignettes let the user see if elements of text are missed or used incorrectly by the classifier, in which case they can exercise caution when considering the prediction. In this way the reviewer determined:
"that some of the descriptions I pay most attention to (the rising PSA values and, to a lesser extent, the high Gleason score) are gray_presumably because the algorithm is ignoring them."

Both PSA and Gleason score are numerical values which are often included in these medical summaries, but which are not captured by our current feature representation. This feedback suggests that an avenue for improved performance would be to incorporate prior clinical knowledge such as the importance of these two scores. Interestingly, it may also improve trust in the system if users could see that the classifier was making use of the elements of the medical summaries that they consider to be most important. The interpretable vignettes also revealed that classification of prostate 
medRxiv preprint doi: https://doi.org/10.1101/2021.07.15.21260082; this version posted July 16, 2021. The copyright holder for this preprint

(which was not certified by peer review) is the author/funder, who has granted medRxiv a license to display the preprint in perpetuity.

It is made available under a CC-BY 4.0 International license Fee-text cause of death predictions

312 cancer death was problematic when negation appeared in the text. Our bag-of-words feature

313 representation would not be expected to handle negation, so the application of methods to detect

314 negation in clinical text data $(37,38)$ would likely boost performance. Off-the-shelf classifiers

315 achieved good performance on the CAP dataset. For different health record datasets, additional effort

316 may be required to achieve sufficient performance for a decision support tool to be useful. The

317 clustering of the health records based on authorship suggested that methods such as multiple-source

318 cross-validation (39) or domain adaptation (40) could be beneficial in dealing with differences in

319 writing styles within other datasets. Other methods to boost performance would likely be task- or

320 domain-specific and could include the addition of numeric clinical features extracted from structured

321 data (41), or the use of state-of-the-art deep learning methods (12,37). Such methods would be

322 compatible with our model-agnostic approach to interpretability.

323 The CAP dataset contains a proxy for 'difficulty' of the cause of death assignment. Although we

324

325

326

327

328

329

330

331

332

333

334

335

336

337

338

339

340

341

342

343

344

345

346

347

348

349

350

351

352

353 were not able to train a model to reliability predict hard cases, our cause-of-death-classifier did show worse performance and calibration on the hard cases than on the easy cases. This suggests that the stratification of cases according to difficulty is meaningful and is likely to have implications for the future development and evaluation of a decision support tool. A systematic investigation of what makes the hard cases more difficult to classify, and which features are most predictive for different types of cases, will help to inform more targeted data acquisition from hospital records. Named-entity recognition approaches could also be adapted to assist with this information retrieval $(12,15)$. Such knowledge could produce significant cost savings in data collection for CAP and similar projects. In practice, the predictions for hard cases are less trustworthy and one way to address this would be to produce reliable estimates of uncertainty (42). In an applied setting it would be important for the transparency of the system to communicate to users the relative risks of both false positive and false negatives.

The feedback of the CAP reviewer has given us confidence in the feasibility of these methods, and the next stage is to develop them into a usable decision support tool, following a user-centric design process with members of the intended user group (43). Key to this will be to adapt the visualisations to be appropriate for users in a clinical setting. We will need to test our classifiers on new CAP reviews to determine how well they generalise to unseen data. Our bag-of-words approach is limited by the size of the training data. There are 1360 words in the test data set that do not appear in our training data (Figure S7 in Online Resource 1), and the CAP dataset has only limited overlap (Figure S8 in Online Resource 1) with an example biomedical corpus (44). To optimise classifier performance in the future will likely require an adapted pre-trained deep learning model (11). We have identified benchmarking datasets (45) that would allow comparison of different classification approaches to ensure that the best model can be selected. It is clear from our results that the different approaches to quantifying feature importance produce distinct feature rankings. Choosing the best approaches to ensure user trust and system transparency will be achieved using A/B testing across a range of users. The continued use of model-agnostic explainability methods will allow abstraction of the decision support interface from the underlying classifier and would allow the tool usable across a range of different tasks and datasets. For example, we plan to test our approach to interpretable document classification on an intensive care dataset (46) that contains free-text medical notes that are routinely used by hospital staff to audit clinical practice.

\section{5. Conclusion}

355 Algorithmic classification of health records, such as the identification of prostate cancer death in the

356 CAP dataset, could reduce the need for complex medical summaries to be reviewed by an 
medRxiv preprint doi: https://doi.org/10.1101/2021.07.15.21260082; this version posted July 16, 2021. The copyright holder for this preprint (which was not certified by peer review) is the author/funder, who has granted medRxiv a license to display the preprint in perpetuity.

It is made available under a CC-BY 4.0 International license Fee-text cause of death predictions

357 independent committee. We have demonstrated use of visual methods to explain classifier

358 predictions to human users, which could be deployed in a future decision support tool to reduce the

359 cognitive burden on individual reviewers. Knowledge of the predictive features could also be used to

360 target data extraction from hospitals, reducing the workload and cost required in creating the free-text

361 summaries. We encourage researchers to take a user-centric approach when developing interpretable

362 machine learning tools, to ensure maximum trust and usability in the system. 
medRxiv preprint doi: https://doi.org/10.1101/2021.07.15.21260082; this version posted July 16, 2021. The copyright holder for this preprint

(which was not certified by peer review) is the author/funder, who has granted medRxiv a license to display the preprint in perpetuity.

It is made available under a CC-BY 4.0 Internatiopal license ree-text cause of death predictions

\section{References}

365

366

367

368

369

370

371

372

373

374

375

376

377

378

379

380

381

382

383

384

385

386

387

388

389

390

391

392

393

394

395

396

397

398

399

1. Demner-Fushman D, Chapman WW, McDonald CJ. What can natural language processing do for clinical decision support? J Biomed Inform. 2009 Oct 1;42(5):760-72.

2. Spasić I, Livsey J, Keane JA, Nenadić G. Text mining of cancer-related information: Review of current status and future directions. Int J Med Inf. 2014 Sep 1;83(9):605-23.

3. Strauss JA, Chao CR, Kwan ML, Ahmed SA, Schottinger JE, Quinn VP. Identifying primary and recurrent cancers using a SAS-based natural language processing algorithm. J Am Med Inform Assoc. 2013 Mar 1;20(2):349-55.

4. Datta MW, Hernandez AM, Schlicht MJ, Kahler AJ, DeGueme AM, Dhir R, et al. Perlecan, a candidate gene for the CAPB locus, regulates prostate cancer cell growth via the Sonic Hedgehog pathway. Mol Cancer. 2006 Mar 1;5(1):9.

5. Banerjee I, Gensheimer MF, Wood DJ, Henry S, Aggarwal S, Chang DT, et al. Probabilistic Prognostic Estimates of Survival in Metastatic Cancer Patients (PPES-Met) Utilizing Free-Text Clinical Narratives. Sci Rep. 2018 Jul 3;8(1):10037.

6. Extracting Cancer Mortality Statistics from Free-text Death Certificates | Proceedings of the 23rd Australasian Document Computing Symposium [Internet]. [cited 2020 Aug 14]. Available from: https://dl.acm.org/doi/abs/10.1145/3291992.3292003

7. Black WC, Haggstrom DA, Gilbert Welch H. All-Cause Mortality in Randomized Trials of Cancer Screening. JNCI J Natl Cancer Inst. 2002 Feb 6;94(3):167-73.

8. Turner EL, Metcalfe C, Donovan JL, Noble S, Sterne JAC, Lane JA, et al. Contemporary accuracy of death certificates for coding prostate cancer as a cause of death: Is reliance on death certification good enough? A comparison with blinded review by an independent cause of death evaluation committee. Br J Cancer. 2016 Jun;115(1):90-4.

9. Devlin J, Chang M-W, Lee K, Toutanova K. BERT: Pre-training of Deep Bidirectional Transformers for Language Understanding. In: NAACL-HLT. 2019.

10. Mujtaba G, Shuib L, Idris N, Hoo WL, Raj RG, Khowaja K, et al. Clinical text classification research trends: Systematic literature review and open issues. Expert Syst Appl. 2019 Feb $1 ; 116: 494-520$.

11. J L, W Y, S K, D K, Ch S, J K. BioBERT: a pre-trained biomedical language representation model for biomedical text mining. Bioinforma Oxf Engl. 2020 Feb 1;36(4):1234-40.

12. Duarte F, Martins B, Pinto CS, Silva MJ. Deep neural models for ICD-10 coding of death certificates and autopsy reports in free-text. J Biomed Inform. 2018 Apr 1;80:64-77.

13. Miftakhutdinov Z, Tutubalina E. KFU at CLEF eHealth 2017 Task 1: ICD-10 Coding of English Death Certificates with Recurrent Neural Networks. 2017.

14. MIMIC-III, a freely accessible critical care database | Scientific Data [Internet]. [cited 2021 Jan 31]. Available from: https://www.nature.com/articles/sdata201635 
medRxiv preprint doi: https://doi.org/10.1101/2021.07.15.21260082; this version posted July 16, 2021. The copyright holder for this preprint (which was not certified by peer review) is the author/funder, who has granted medRxiv a license to display the preprint in perpetuity. It is made available under a CC-BY 4.0 International license Fee-text cause of death predictions

400

401

402

403

404

405

406

407

408

409

410

411

412

413

414

415

416

417

418

419

420

421

422

423

424

425

426

427

428

429

430

431

432

433

434

435

436

15. Mujtaba G, Shuib L, Raj RG, Rajandram R, Shaikh K. Automatic Text Classification of ICD-10 Related CoD from Complex and Free Text Forensic Autopsy Reports. In: 2016 15th IEEE International Conference on Machine Learning and Applications (ICMLA). 2016. p. 1055-8.

16. Van Mulligen EM, Afzal Z, Akhondi S, Vo D, Kors J. Erasmus MC at CLEF eHealth 2016: Concept recognition and coding in French texts. 2016 Jan 1 [cited 2021 Jan 31]; Available from: https://repub.eur.nl/pub/100036/

17. Cabot C, Soualmia L, Dahamna B, Darmoni S. SIBM at CLEF eHealth Evaluation Lab 2016: Extracting Concepts in French Medical Texts with ECMT and CIMIND. In 2016.

18. Carvalho DV, Pereira EM, Cardoso JS. Machine Learning Interpretability: A Survey on Methods and Metrics. Electronics. 2019 Aug;8(8):832.

19. Binns R. Fairness in Machine Learning: Lessons from Political Philosophy. In: Conference on Fairness, Accountability and Transparency [Internet]. 2018 [cited 2020 Aug 16]. p. 149-59. Available from: http://proceedings.mlr.press/v81/binns18a.html

20. Diakopoulos N. Accountability in algorithmic decision making. Commun ACM. 2016 Jan 25;59(2):56-62.

21. de Laat PB. Algorithmic Decision-Making Based on Machine Learning from Big Data: Can Transparency Restore Accountability? Philos Technol. 2018 Dec;31(4):525-41.

22. Sokol K, Hepburn A, Poyiadzi R, Clifford M, Santos-Rodriguez R, Flach P. FAT Forensics: A Python Toolbox for Implementing andDeploying Fairness, Accountability and

TransparencyAlgorithms in Predictive Systems. J Open Source Softw. 2020 May 19;5(49):1904.

23. treeinterpreter: Package for interpreting scikit-learn's decision tree and random forest predictions. [Internet]. [cited 2020 Sep 11]. Available from: https://github.com/andosa/treeinterpreter

24. Archer KJ, Kimes RV. Empirical characterization of random forest variable importance measures. Comput Stat Data Anal. 2008 Jan 10;52(4):2249-60.

25. Ribeiro MT, Singh S, Guestrin C. "Why Should I Trust You?”: Explaining the Predictions of Any Classifier. In: Proceedings of the 22nd ACM SIGKDD International Conference on Knowledge Discovery and Data Mining [Internet]. New York, NY, USA: Association for Computing Machinery; 2016 [cited 2020 Sep 11]. p. 1135-44. (KDD '16). Available from: https://doi.org/10.1145/2939672.2939778

26. Lundberg SM, Lee S-I. A Unified Approach to Interpreting Model Predictions. Adv Neural Inf Process Syst. 2017;30:4765-74.

27. Niculescu-Mizil A, Caruana R. Predicting good probabilities with supervised learning. In: Proceedings of the 22nd international conference on Machine learning [Internet]. New York, NY, USA: Association for Computing Machinery; 2005 [cited 2020 Sep 12]. p. 625-32. (ICML '05). Available from: https://doi.org/10.1145/1102351.1102430

28. Amrit C, Paauw T, Aly R, Lavric M. Identifying child abuse through text mining and machine learning. Expert Syst Appl. 2017 Dec 1;88:402-18.

This is a provisional file, not the final typeset article 
medRxiv preprint doi: https://doi.org/10.1101/2021.07.15.21260082; this version posted July 16, 2021. The copyright holder for this preprint

(which was not certified by peer review) is the author/funder, who has granted medRxiv a license to display the preprint in perpetuity.

It is made available under a CC-BY 4.0 Internatiopal licensee ree-text cause of death predictions

437

438

439

440

441

442

443

444

445

446

447

448

449

450

451

452

453

454

455

456

457

458

459

460

461

462

463

464

465

466

467

468

469

470

471

472

473

474

29. Atutxa A, de Ilarraza AD, Gojenola K, Oronoz M, Perez-de-Viñaspre O. Interpretable deep learning to map diagnostic texts to ICD-10 codes. Int J Med Inf. 2019 Sep 1;129:49-59.

30. Stiglic G, Kocbek P, Fijacko N, Zitnik M, Verbert K, Cilar L. Interpretability of machine learning-based prediction models in healthcare. WIREs Data Min Knowl Discov. 2020;10(5):e1379.

31. Berge GT, Granmo O, Tveit TO, Goodwin M, Jiao L, Matheussen BV. Using the Tsetlin Machine to Learn Human-Interpretable Rules for High-Accuracy Text Categorization With Medical Applications. IEEE Access. 2019;7:115134-46.

32. Lipton ZC. In machine learning, the concept of interpretability is both important and slippery. Mach Learn. :28.

33. Chawla P, Hazarika S, Shen H-W. Token-wise sentiment decomposition for ConvNet: Visualizing a sentiment classifier. Vis Inform. 2020 Jun 1;4(2):132-41.

34. "What is relevant in a text document?": An interpretable machine learning approach [Internet]. [cited 2021 Mar 30]. Available from:

https://journals.plos.org/plosone/article?id=10.1371/journal.pone.0181142

35. Ce P, Tie B. An Analysis Method for Interpretability of CNN Text Classification Model. Future Internet. 2020 Dec;12(12):228.

36. Hamdy FC, Donovan JL, Lane JA, Mason M, Metcalfe C, Holding P, et al. 10-Year Outcomes after Monitoring, Surgery, or Radiotherapy for Localized Prostate Cancer. N Engl J Med. 2016 Oct 13;375(15):1415-24.

37. Chen L. Attention-Based Deep Learning System for Negation and Assertion Detection in Clinical Notes [Internet]. Rochester, NY: Social Science Research Network; 2019 Jan [cited 2020 Aug 18]. Report No.: ID 3342402. Available from: https://papers.ssrn.com/abstract=3342402

38. Miller T, Bethard S, Amiri H, Savova G. Unsupervised Domain Adaptation for Clinical Negation Detection. In: BioNLP 2017 [Internet]. Vancouver, Canada,: Association for Computational Linguistics; 2017 [cited 2020 Aug 18]. p. 165-70. Available from: https://www.aclweb.org/anthology/W17-2320

39. McWilliams CJ, Lawson DJ, Santos-Rodriguez R, Gilchrist ID, Champneys A, Gould TH, et al. Towards a decision support tool for intensive care discharge: machine learning algorithm development using electronic healthcare data from MIMIC-III and Bristol, UK. BMJ Open. 2019 Mar 1;9(3):e025925.

40. Tzeng E, Hoffman J, Saenko K, Darrell T. Adversarial Discriminative Domain Adaptation. In 2017 [cited 2020 Aug 18]. p. 7167-76. Available from: https://openaccess.thecvf.com/content_cvpr_2017/html/Tzeng_Adversarial_Discriminative_Dom ain_CVPR_2017_paper.html

41. Apostolova E, Wang T, Tschampel T, Koutroulis I, Velez T. Combining Structured and Free-text Electronic Medical Record Data for Real-time Clinical Decision Support. In: Proceedings of the 18th BioNLP Workshop and Shared Task [Internet]. Florence, Italy: Association for 
medRxiv preprint doi: https://doi.org/10.1101/2021.07.15.21260082; this version posted July 16, 2021. The copyright holder for this preprint (which was not certified by peer review) is the author/funder, who has granted medRxiv a license to display the preprint in perpetuity. It is made available under a CC-BY 4.0 International license Free-text cause of death predictions

Computational Linguistics; 2019 [cited 2020 Aug 14]. p. 66-70. Available from: https://www.aclweb.org/anthology/W19-5007

42. Gal Y, Ghahramani Z. Dropout as a Bayesian Approximation: Representing Model Uncertainty in Deep Learning. :10.

43. Bezemer T, Groot MCH de, Blasse E, Berg MJ ten, Kappen TH, Bredenoord AL, et al. A Human(e) Factor in Clinical Decision Support Systems. J Med Internet Res. 2019;21(3):e11732.

44. Krallinger M, Akhondi S. Overview of the Chemical- Protein relation extraction track. :32.

45. Peng Y, Yan S, Lu Z. Transfer Learning in Biomedical Natural Language Processing: An Evaluation of BERT and ELMo on Ten Benchmarking Datasets. In: Proceedings of the 18th BioNLP Workshop and Shared Task [Internet]. Florence, Italy: Association for Computational Linguistics; 2019 [cited 2021 Feb 1]. p. 58-65. Available from: https://www.aclweb.org/anthology/W19-5006

46. McWilliams C, Inoue J, Wadey P, Palmer G, Santos-Rodriguez R, Bourdeaux C. Curation of an intensive care research dataset from routinely collected patient data in an NHS trust. F1000Research [Internet]. 2019 Aug 19 [cited 2021 Feb 1];8. Available from: https://www.ncbi.nlm.nih.gov/pmc/articles/PMC6733376/ 
medRxiv preprint doi: https://doi.org/10.1101/2021.07.15.21260082; this version posted July 16, 2021. The copyright holder for this preprint (which was not certified by peer review) is the author/funder, who has granted medRxiv a license to display the preprint in perpetuity. It is made available under a CC-BY 4.0 International license .

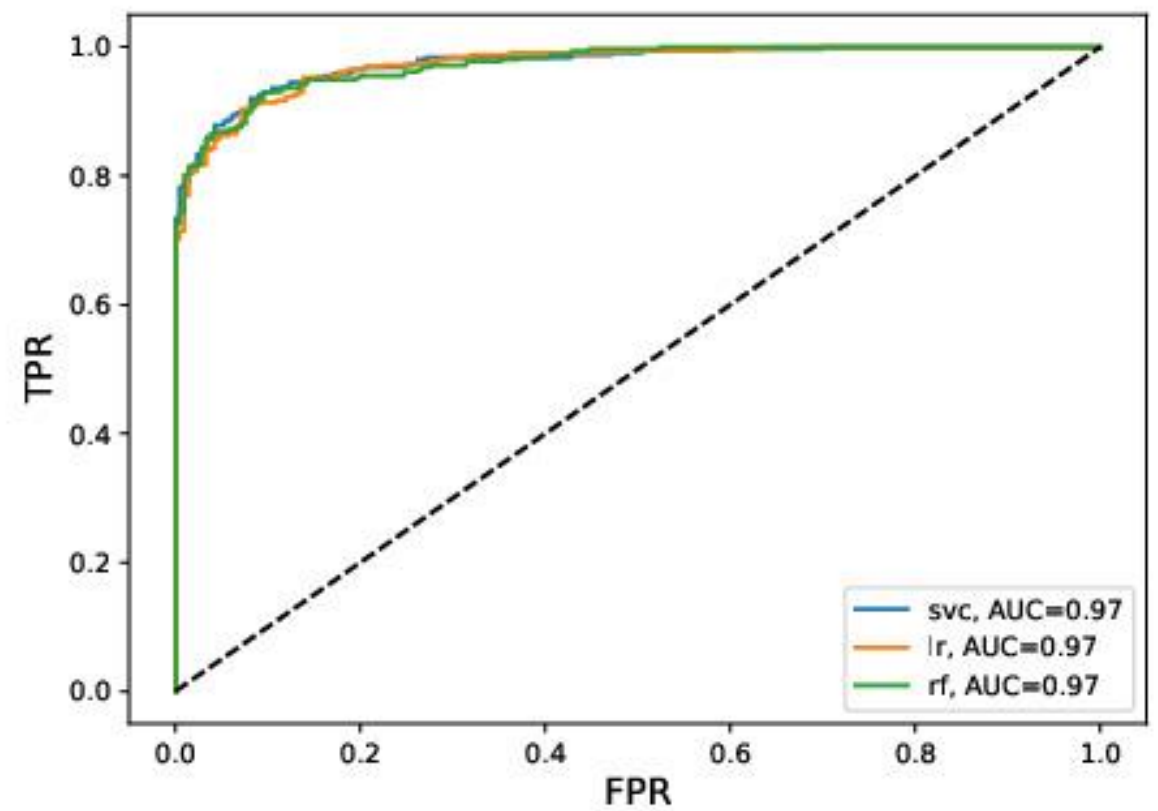

Figure 1: Receiver operating characteristic (ROC) curve for three classifiers predicting cause of death from the CAP dataset, plotting the true positive rate (TPR) against the false positive rate (FPR). Performance curves are shown for: $S V C=$ Support vector classifier; $L R=$ logistic regression; $R F=$ random forest
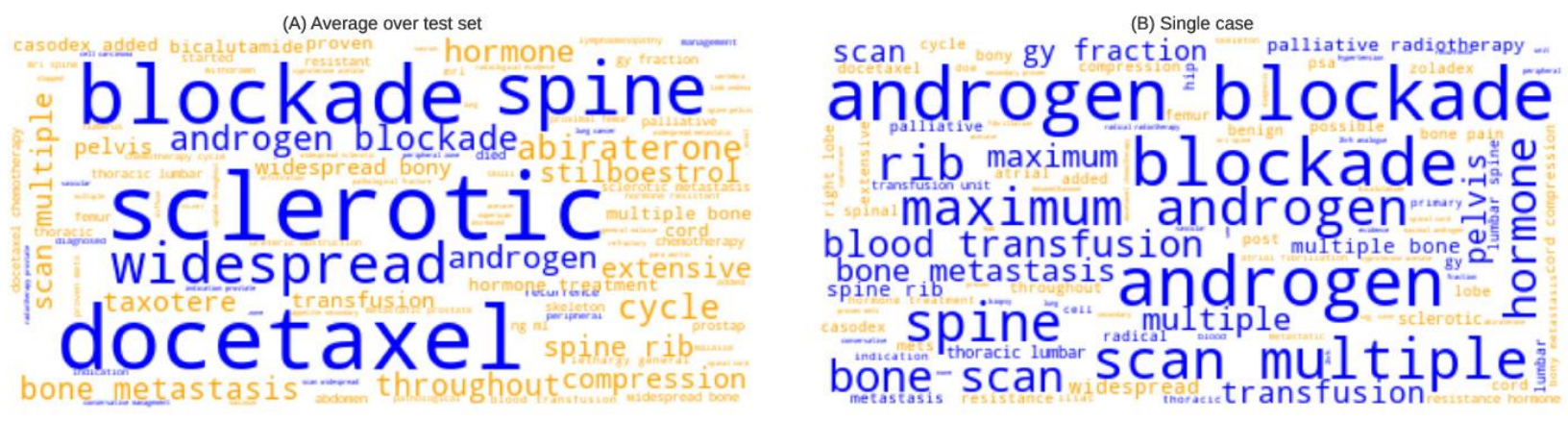

Figure 2: Word clouds indicating the feature contributions towards random forest predictions of prostate cancer death (for the classifier depicted in Figure 1). The size of the word or bigram indicates its relative importance. Blue words or bigrams are indicative of prostate cancer death, while orange is indicative of not prostate cancer death. Feature contributions determined using TreeInterpreter (see main text). (A) Average feature contributions over the CAP test set; (B) a single case that was correctly predicted prostate cancer death by the classifier (shown in Figure 3) 
medRxiv preprint doi: https://doi.org/10.1101/2021.07.15.21260082; this version posted July 16, 2021. The copyright holder for this preprint (which was not certified by peer review) is the author/funder, who has granted medRxiv a license to display the preprint in perpetuity.

It is made available under a CC-BY 4.0 International license Free-text cause of death predictions

Interpretable Vignette Classification for Cause of Death Review

\begin{tabular}{l} 
Study ID number : \\
Date of death : \\
$\begin{array}{l}\text { Date of diagnosis : } \\
\text { Actual death code: } 2\end{array}$ \\
\hline
\end{tabular}

Feature contribution legend

\begin{tabular}{lll} 
bone scan & hptake right lesion & hormone \\
Largest negative contribution & smallest contributions & Largest positive contribution \\
\hline
\end{tabular}

Clinical features at diagnosis

Gleason Score at diagnosis (with dates): 2010 ? Gleason 3+5=8

Clinical stage (TNM): $\square / 2009$ ? ?Benign?, TRus volume $=60 \mathrm{cc}$

Pathological stage (TNM):

Figure 3: Snapshot of an 'interpretable vignette' that allows users to engage with the prediction that is made by the classifier. This case was correctly predicted to be a prostate cancer death by the classifier (cause of death code $=2$ ). As in figure 2 the word (or bigram) size indicates the magnitude of the contribution of that feature to the prediction and the colour indicates the sign of the contribution. Here the original format of the vignettes is retained, which is the format in which the decision makers would normally engage with the document. Full interpretable vignette examples are provided in Online Resources 2 and 3

This is a provisional file, not the final typeset article 